\title{
Design and Performance Analysis of Log Periodic Dipole Antenna at Uhf Band.
}

\author{
${ }^{1}$ Ogherohwo E. P., ${ }^{2}$ Adeniran A. O. \\ ${ }^{1}$ Department of Physics, University of Jos, Jos, Plateau State, Nigeria. \\ ${ }^{2}$ Department of Pure and Applied Physics, Ladoke Akintola University of Technology, P.M.B 4000, Ogbomoso, \\ Oyo State, Nigeria.
}

Abstract: The paper described the design and performance analysis of log periodic dipole antenna at frequency $1800 \mathrm{MHz}-2500 \mathrm{MHz}$ (Ultra High Frequency Band), the antenna has been modeled using ADS layout and the S parameter was presented. A good impedance match in a wide frequency range has been achieved by a suitable choice of the antenna feeder impedance. The radiation pattern, Directivity, Return loss, Voltage Standing Wave Ratio (VSWR) and Gain are in accordance with the desired valuesare presented.

Keywords:LPDA (Log Periodic Diploe Antenna), Agilent.

\section{Introduction}

The design of indoor coverage systems for mobile and wireless communications has tended to join services working at different frequencies into one distribution system.it reduces the number of used components but requires extended frequency range from them. Therefore, a demand for antennas that would cover joint bands has arisen. For broadband applications, the Log Periodic Dipole Antenna (LPDA) type has been commonly used. Its advantage is that within the design band its performance isessentially frequency independent, including radiation resistance (hence VSWR) and radiation pattern (gain and front to back ratio).

\section{II.BasicTheory of LPDA}

The LPDA is frequency independent in that the electrical properties such as the mean resistance level $R_{o}$, characteristic impedance of the feed line $Z_{o}$, and driving point admittance $Y_{o}$, vary periodically with the logarithm of the frequency. As the frequency $f_{1}$ is shifted to another frequency $f_{2}$ within the passband of the antenna, the relationship is $f_{1}=\frac{f_{1}}{\tau}$, where

$\mathrm{T}=\mathrm{a}$ design parameter, aconstant; $\tau<1.0$.Also

$f_{n}=\frac{f_{1}}{\tau^{n-1}}$

$f_{1}=$ lowestfrequenccy

$f_{n}=$ highestfrequeency

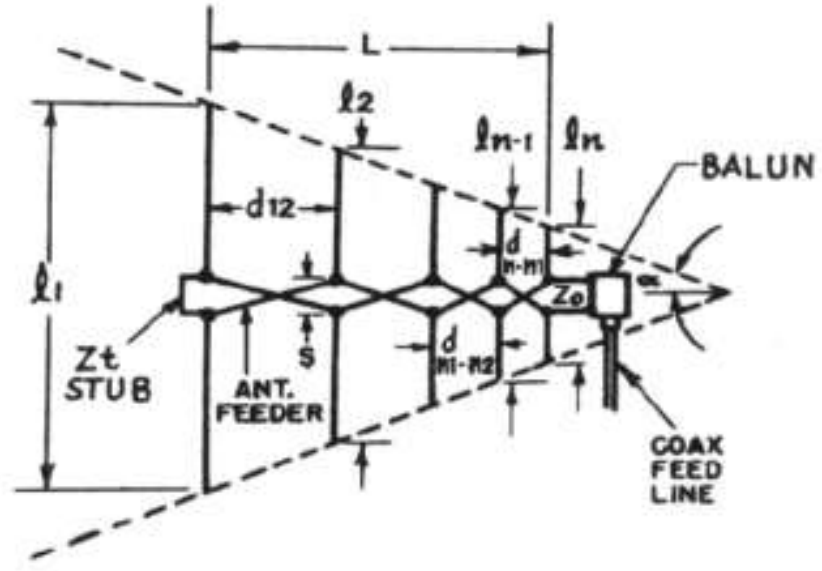

Figure 1: Log Periodic Dipole Antenna 


\section{III.Design Steps}

The basic arrangement of a log periodic array excited by a two wire line (antenna feeder) is shown in the figure 1 along with the geometry defining formulars. The array are dipole antennas excited with 180 phase shift ;their length decreases according to [3] and [4], respectively, where $\tau<1$ is a design constant called periodicity. Relative spacing oof the elements is defined by [4],the two element supporting booms act as twin line feeder; the required 180 phase shift is implemented by attaching the elements alternately to the first and second boom(Fig 2).

Formulars and graphs published in [3] and [4] have been used for the antenna design which explains step by step design procedures. The design input parameters are the nominal input resistance $R_{o}$, the desired gain $\mathrm{G}$ relative to isotropic radiator, and frequency range expressed as the lower $\left(f_{1}\right)$ and upper $\left(f_{2}\right)$ operating frequencies. The relative antenna bandwidth is

$$
B=\frac{f_{2}}{f_{1}} \text {. }
$$

In our case $R_{o}=50 \Omega$ this was considered so as to achieve a ofVSWR $<1.5$, Gain=9.5dBi.

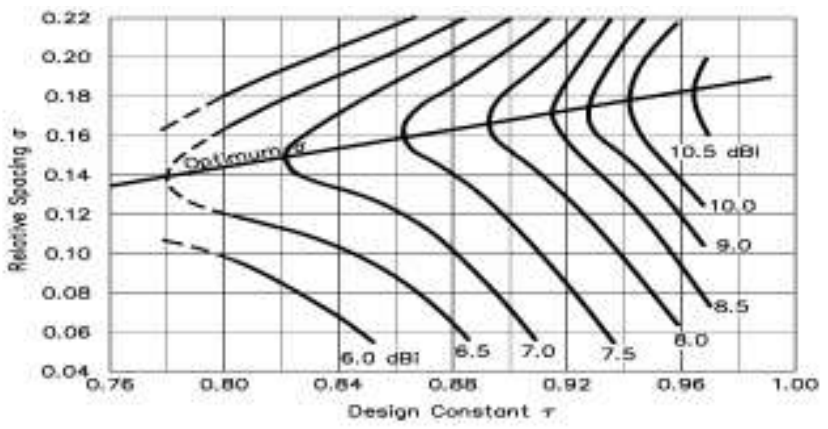

Figure 2:LPDA gain as a function of $\tau$ and $\sigma$

Step 1: Using Figure (1), find $\tau$ and the figure(2) is labeled by gain .Determine the curve corresponding to the desired gain $\mathrm{G}$ and find its intersection with the straight line.

$$
\sigma=0.243 \tau-0.051
$$

The intersection defines the sought $\tau$ and $\sigma$.

Step 2: By successive application of (4) to (6) find the active region bandwidth $B_{a r}$, structure bandwidth $B_{s}$ and the number of required dipole elements Roundoff to the nearest higher integer.

Step 3: Determine the element lengths and spacing, Using (3) and (7), find the length $L_{1}$ of the longest dipole and the spacing $R_{1}-R_{2}$ between the two longest dipoles. Then, using [3] and [4],find the lengths and Spacing of the remaining elements.

$$
\begin{aligned}
& B_{a r}=1.1+7.7(1-\tau)^{2} \frac{4 \sigma}{1-\tau} \\
& B_{s}=B \cdot B_{a r}=\frac{f_{i}}{f_{1}} B_{a r} \\
& N=1+\frac{\log B_{s}}{\log \frac{1}{\tau}} \\
& L_{1}=\frac{1}{2} \cdot \frac{3 \cdot 10^{8}}{f_{1}} \\
& R_{1}-R_{2}=\frac{L_{1}-L_{2}}{2} \cdot \frac{4 \sigma}{1-\tau}
\end{aligned}
$$


Design and Performance Analysis of Log Periodic Dipole Antenna at UHF Band.

Step 4:Determine the short position, if higher front back ratio at the lowest frequency is desired, the antenna feeder should be shorted at a distance $L_{s}$ behind the longest element. The short acts as a reflector; its distance from the longest element should be $L_{s}=\frac{L_{1}}{4}$.

The antenna parameters obtained in accordance with the described steps are

$$
(\tau)=0.930, \sigma=0.715, B_{a r}=1.5203, B_{s}=1.9565, \mathrm{~N}=10.236, L_{s}=20.83 \mathrm{~mm}
$$

The dipole lengths and spacing's in $\mathrm{mm}$ are summarized in the following table (1).

Table (1): Showing the Dipole elements lengths and spacing.

\begin{tabular}{|l|l|l|l|l|l|l|l|l|l|l|}
\hline Elements (i) & 1 & 2 & 3 & 4 & 5 & 6 & 7 & 8 & 9 & 10 \\
\hline$L_{i}$ & 83.3 & 77.50 & 72.05 & 67.00 & 62.31 & 57.95 & 53.89 & 50.12 & 46.60 & 43.40 \\
\hline$R_{i}-R_{i-1}$ & 29.2 & 27.1 & 25.2 & 23.5 & 21.8 & 20.3 & 18.9 & 17.5 & 16.3 & ------ \\
\hline
\end{tabular}

\section{IV.Realization and Analysis}

An aluminum tube with outer diameter $2 \mathrm{a}=4 \mathrm{~mm}$ was used for all the antenna dipole elements. Using the same diameter is not optimum with respect to VSWRrather; the length to diameter ratio (slimness factor) $S=\frac{L_{1}}{(2 a)}$ should be kept constant.

A pair of aluminum tubes with outer diameter $b=5.5 \mathrm{~mm}$ was used as the feeder .A $50 \Omega$ semi flexible coaxial cable was inserted into one of the feeder tubes. On the long dipole end, the cable was terminated by an $\mathrm{N}$-female connector .At the other (feed point) end, the semi flex braiding was soldered to the feeder tube it was inserted in; the inner conductor was soldered to the opposite feeder tube ,as seen in the Figure (1).the distance between the tubes should be

$d_{f}=b \cosh \left(Z_{o} / 120\right)$

Where $Z_{o}=$ feeder characteristics impedance ensuring the lowest possible VSWR [1]:

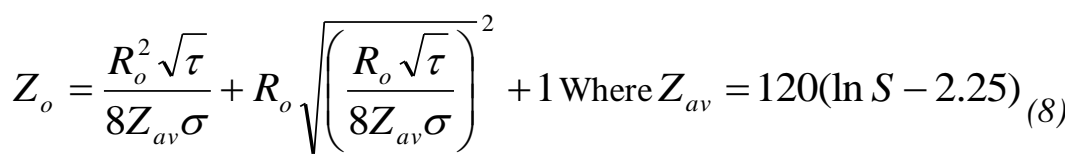

And $\mathrm{S}$ is the average slimness factor of the elements. The formula(for $\mathrm{S}=20.83$ ) led to the value $Z_{o}=68.5 \Omega$ and $d_{f}=5.86 \mathrm{~mm}$. Teflon spacers were used to keep the feeder tubes at the designed distance.

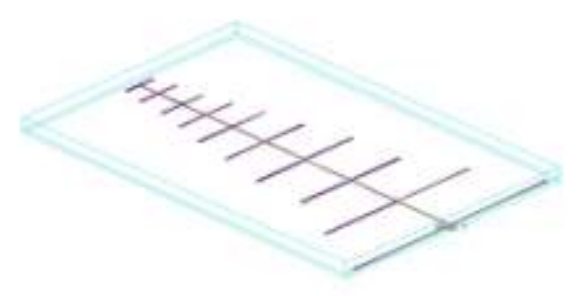

Figure 3: LPDA layout on Agilent at free space

\section{V.Results and Discussions}

The simulated step response, VSWR, Return loss ,radiation pattern (Electric and Magnetic), Radiated power, Output impedance Gain and Directivity are shown in the Figures $(4,5,6,7$,and 8 ). The antenna was simulated using Agilent software layout for momentum simulation and it was consider under free space. The return loss was $-0.013 \mathrm{~dB}$ as shown in Figure: 4, both $\mathrm{E}$-plane and H-plane patterns were measured at angular in a wider frequency of $1800 \mathrm{MHz}-2500 \mathrm{MHz}$, step $10 \mathrm{MHz}) .137$ relative E-plane patterns was obtained as presented in Figure 7 and 9, Directivity was $5.19 \mathrm{~dB}$ with simulated gain of $9.45 \mathrm{~dB}$ and the maximum output radiated power in Figure: 9 was obtained to be $2.68 \mathrm{E}-09$. 

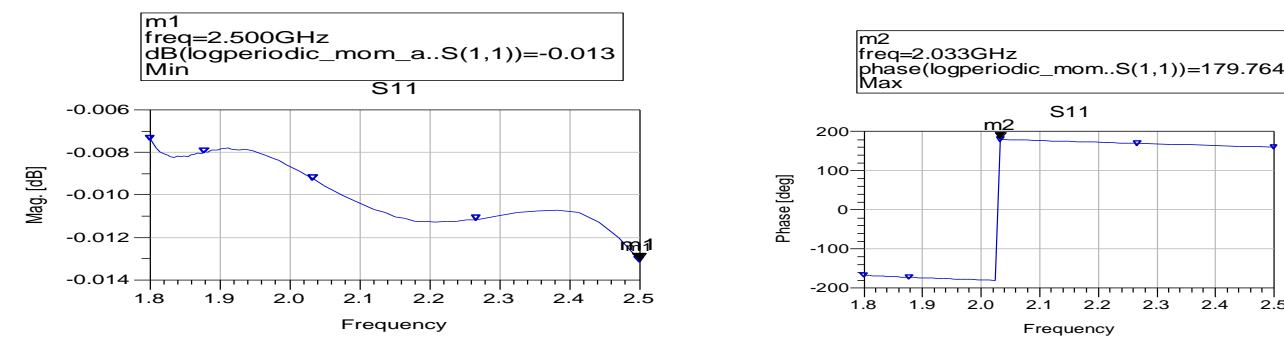

Figure 4: The input and output return loss for LPDA
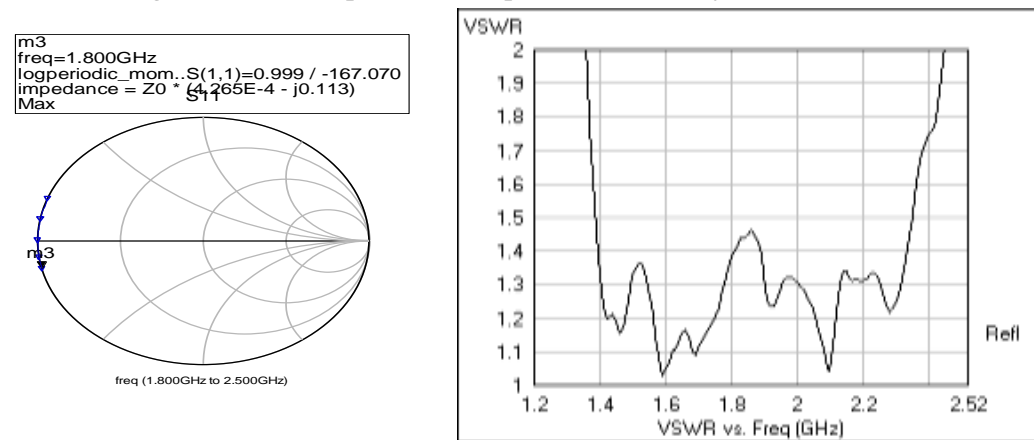

Figure 5: The Output impedance for LPDAFigure 6: Response VSWR of the LPDA
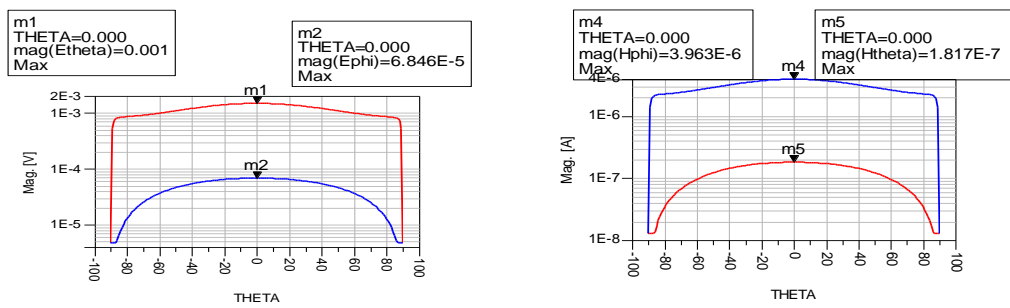

Figure 7: 2D Radiation pattern $E$ (Electric) and $H$ (Magnetic)

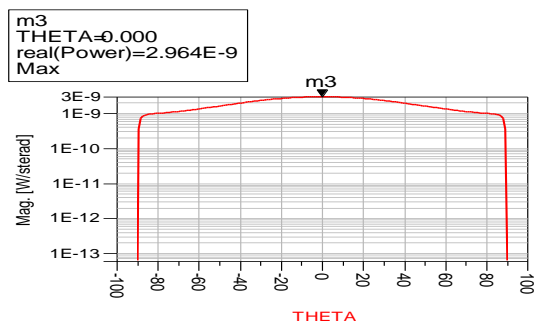

Figure 8:Output Radiated powerFigure 9:3D radiation pattern

\section{Conclusion}

The design and simulated performance analysis of the Gain, Return loss, radiation pattern and power radiated of log Periodic Dipole Antenna at $1800 \mathrm{MHz}-2500 \mathrm{MHz}$ has been presented. From the whole band the simulated parameters was in agreement with the desired value of the design.

\section{References}

[1] Agilent Software Inc.(Advance Design System 2009).

[2] Frantisek, H.Jan.B, Vladimir. "Design and investigation of a Log Periodic Antenna for DCS,PCS and UMTS mobile Communications Band"

[3] Nowwatzky, D. Logarithimisch periodische Antennen.Technische Milleilungen des RFZ, Jahrg.7/Heft2, June 1963, pp.77-80, and Jahrg,7/Heft3,Sept.1963,pp.127-133.(http://home.t-online.de/home/Dieter.Nowatzky/doc.htm)

[4] Severns,R.,Beezly,B.,Hare,E.,Log Periodic Arrays .In the ARRL.Antenna Book[CD-ROM].The American Radio Relay League,Inc.Newington,CT06111-1494.

[5] Banic ,B.,Hajach,P.,'Design and Simulation of Properties of Log Periodic Dipole Antenna.In Radioelekrionika 2000,Brastslava,1216,Sept,20000,pp108-109. 